\title{
Vinyldiazolactone as a Vinylcarbene Precursor: Highly Selective C-H Insertion and Cyclopropanation Reaction
}

\author{
Darren Bykowski, Kou-Hui Wu, and Michael P. Doyle* \\ Department of Chemistry, University of Maryland, College Park, Maryland
}

\section{Supporting Information}

\section{Table of Contents}

General Information

Preparation of 1

Table 1 Experimental

Table 2 Experimental

Synthesis of 4

Determination of Absolute Stereochemistry of 2a

Determination of Relative Stereochemistries of $\mathbf{2 b}$, 3a

${ }^{1} \mathrm{H}$ NMR Spectra

${ }^{13} \mathrm{C}$ NMR Spectra

HSQC Spectrum of $\mathbf{2 b}$
Page

S2

S2

S2

S3

S4

S5

S6

S8

S17

S26 
General Information: NMR spectra were recorded on a Bruker DRX-400 $\mathrm{MHz}$ instrument in a solution of deuterochloroform unless otherwise noted. Chemical shifts of ${ }^{1} \mathrm{H}$ NMR are quoted relative to internal $\mathrm{Me}_{4} \mathrm{Si}(0.00 \mathrm{ppm})$, those of ${ }^{13} \mathrm{C}$ NMR are quoted relative to residual solvent (77.0 ppm). NMR spectra of $\mathbf{1}$ were obtained using deuterated dichloromethane, chemical shifts of the ${ }^{1} \mathrm{H}$ NMR are quoted relative to residual solvent (5.32 ppm), chemical shifts of the ${ }^{13} \mathrm{C}$ are quoted relative to residual solvent (54.0 ppm). High-resolution spectra were obtained on a JEOL SX102a spectrometer. Thin layer chromatography was performed on Merck Silica Gel $40 \mathrm{~F}_{254}$ glass backed plates, visualization was achieved with UV or $\mathrm{KMnO}_{4}$ stain. Column chromatography was performed on 40-63 $\mu \mathrm{m}, 230-400$ mesh, $60 \mathrm{~A}$ silica gel. Cyclopropanation substrates 1vinylcyclohexene, ${ }^{1}$ 1-phenylbutadiene, ${ }^{2}$ 1-phenyl-3-methylbutadiene were prepared according to literature procedures, all other reagents were purchased from Aldrich. Anhydrous dichloromethane and tetrahydrofuran were obtained by dried by nitrogen forced-flow over activated alumina as described by Grubbs. ${ }^{3}$

Preparation of 1: A solution of 3,5-dihydro-pyran-2-one (400 mg, $4.10 \mathrm{mmol}$ ) in acetonitrile $(40 \mathrm{~mL})$ was stirred at $0{ }^{\circ} \mathrm{C}$. 4-Acetamidobenzenesulfonyl azide $(1.23 \mathrm{~g}, 5.10$ $\mathrm{mmol})$ and $\mathrm{DBU}(0.77 \mathrm{~mL}, 5.1 \mathrm{mmol})$ were added to the stirred solution. After $3 \mathrm{~h}$, the resulting brown mixture was evaporated to a thick oil. The mixture was purified via flash silica gel chromatography (2:1 hexanes/ethyl acetate, $\mathrm{Rf}=0.47)$, eluting 1 as a bright yellow band. Fractions were evaporated and dried under vacuum to yield $\mathbf{1}$ as an orange solid (237 mg, 47\%): mp $60{ }^{\circ} \mathrm{C}$; ${ }^{1} \mathrm{H}$ NMR (400 MHz, $\left.\mathrm{CD}_{2} \mathrm{Cl}_{2}\right) \delta 6.20$ (dt, $J=10.0,1.8$ $\mathrm{Hz}, 1 \mathrm{H}), 5.38(\mathrm{td}, J=3.3,10.0 \mathrm{~Hz}, 1 \mathrm{H}), 4.99$ (dd, $J=3.3,1.8 \mathrm{~Hz}, 2 \mathrm{H}) ;{ }^{13} \mathrm{C}$ NMR $(100$ $\mathrm{MHz}, \mathrm{CD}_{2} \mathrm{Cl}_{2}$ ) $\delta$ 164.5, 114.5, 113.5, 70.5 (C = $\mathrm{N}_{2}$ missing); IR (neat) 3073, 2882, 2098, $1668 \mathrm{~cm}^{-1}$; HRMS (EI) calcd for $\mathrm{C}_{5} \mathrm{H}_{4} \mathrm{~N}_{2} \mathrm{O}_{2}$ 124.0273, found 124.0275 (M+).

General Procedure for Table 1: To a flame dried flask containing anhydrous dichloromethane $(20 \mathrm{~mL})$, dirhodium catalyst $(.013 \mathrm{mmol})$, and 1,4-cyclohexadiene (0.61 $\mathrm{mL}, 6.5 \mathrm{mmol}$ ) were added. The apparatus was degassed and heated to reflux under nitrogen. A solution of $\mathbf{1}$ (160 mg, $1.29 \mathrm{mmol}$ in $10 \mathrm{~mL}$ dichloromethane) was added over $8 \mathrm{~h}$. Upon completion of addition, the reaction mixture was refluxed for an additional $1 \mathrm{~h}$, then filtered through a short silica gel plug to remove the dirhodium catalyst. Integration of diagnostic ${ }^{1} \mathrm{H}$ NMR signals of $\mathbf{2 a}(4.80 \mathrm{ppm})$ and $\mathbf{2 b}(4.98 \mathrm{ppm})$ were used to determine relative ratios of $\mathbf{2 a}, \mathbf{b}$. GC was used to determine the enantiomeric excess of 2a prior to column chromatographic purification. Silica gel column chromatography (3:1 hexanes/ethyl acetate $\mathrm{Rf}=0.40$ ) of the reaction mixture provided an unseparated mixture of 2a,b from which isolated yields were calculated from the mass of the product. Pure samples of $\mathbf{2 a}$ and $\mathbf{2 b}$ were obtained by subsequent silica gel chromatography (10:1 hexane/ethyl acetate), but only partial separation was achieved.

2a (from Table 1, entry 6): Clear oil, GC: Chiraldex G-TA (30m x $0.25 \mathrm{~mm}$ ), $140{ }^{\circ} \mathrm{C} / 10$ $\min , 1{ }^{\circ} \mathrm{C} / \mathrm{min}$ ramp to $160{ }^{\circ} \mathrm{C}$ ), retention times $26.2 \mathrm{~min}(R)$ and $29.2 \mathrm{~min}(S), 80 \%$ ee $R$; $[\alpha]_{\mathrm{D}}{ }^{21}+79.5^{\circ}\left(c=0.31, \mathrm{CHCl}_{3}\right) .{ }^{1} \mathrm{H}$ NMR $\left(400 \mathrm{MHz}, \mathrm{CDCl}_{3}\right) \delta$ 5.97-5.76 (comp, 4H),

\footnotetext{
${ }^{1}$ Herz, W.; Juo, R.-R. J. Org. Chem. 1985, 50, 618.

${ }^{2}$ Okamoto, T.; Kobayashi, K.; Oka, S.; Tanimoto, S. J. Org. Chem. 1988, 53, 4897.

${ }^{3}$ Pangborn, A. B.; Giardello, M. A.; Grubbs, R. H.; Rosen, R. K.; Timmers, F. J. Organometallics 1996, 15, 1518.
} 
5.65-5.50 (comp, 2H), 4.83-4.78 (comp, 2H), 3.62-3.55 (m, 1H), 3.09-3.04 (m, 1H), 2.642.58 (comp, $2 \mathrm{H}) ;{ }^{13} \mathrm{C}$ NMR (100 MHz, $\left.\mathrm{CDCl}_{3}\right)$ 170.6, 127.5, 127.1, 125.87, 123.9, 124.0, 122.9, 68.8, 44.4, 38.1, 26.2; IR (neat) 3018, $1733 \mathrm{~cm}^{-1}$; HRMS (EI) calcd for $\mathrm{C}_{11} \mathrm{H}_{12} \mathrm{O}_{2}$ 176.0837, found $176.0835(\mathrm{M}+)$.

2 b (from Table 1, entry 2): Clear oil, ${ }^{1} \mathrm{H}$ NMR (400 MHz, $\left.\mathrm{CDCl}_{3}\right) \delta 5.95$ (dt, $J=10.5$, $2.9 \mathrm{~Hz}, 1 \mathrm{H}), 5.63$ (m, 2H), 5.42 (dt, $J=10.5,1.9 \mathrm{~Hz}, 1 \mathrm{H}), 4.98$ (dd, $J=2.9,1.9 \mathrm{~Hz}, 2 \mathrm{H}$ ), 2.54-2.42 (comp, 2H), 2.19-2.16 (m, 2H), 2.09-2.01 (m 2H); ${ }^{13} \mathrm{C}$ NMR (100 MHz, $\left.\mathrm{CDCl}_{3}\right) \delta 172.5,124.2,122.9,121.1,68.5,28.0,26.1,19.6$; IR (neat) 2088, $1712 \mathrm{~cm}^{-1}$; HRMS (EI) calcd for $\mathrm{C}_{11} \mathrm{H}_{12} \mathrm{O}_{2}$ 176.0837, found 176.0837 (M+).

General Procedure for Table 2: To a flame dried flask containing anhydrous dichloromethane $(20 \mathrm{~mL}), \mathrm{Rh}_{2}(S, R$-MenthAZ) $(17 \mathrm{mg}, 0.013 \mathrm{mmol})$ and olefin (3.3 mmol) were added. The apparatus was degassed and heated to reflux under nitrogen. A solution of 1 (160 mg, $1.29 \mathrm{mmol}$ in $10 \mathrm{~mL}$ dichloromethane) was added over $8 \mathrm{~h}$. Upon completion of addition, the reaction mixture was refluxed for a further $1 \mathrm{~h}$, then filtered through a short silica gel plug to remove the dirhodium catalyst. Analysis by ${ }^{1} \mathrm{H}$ NMR was used to determine the ratios of $E$ - and Z-cyclopropane isomers. Enantiomeric excess was determined by GC or HPLC, as indicated. Isolated yields of 3a-d were determined after purification by silica gel column chromatography.

3a: $(6: 1$ hexanes/EtOAc, $\mathrm{Rf}=0.22) 74 \%$, white solid, mp $98{ }^{\circ} \mathrm{C}$; GC: $(\mathrm{B}-\mathrm{DM}, 30 \mathrm{~m} \mathrm{x}$ $0.25 \mathrm{~mm} ; 0.25 \mu \mathrm{m}$ film, $140{ }^{\circ} \mathrm{C} / 10 \mathrm{~min}, 1{ }^{\circ} \mathrm{C} / \mathrm{min}$ ramp to $160{ }^{\circ} \mathrm{C}$ ), retention times of 45.3 min (minor) and $47.3 \mathrm{~min}$ (major), 84\% ee; $[\alpha]_{\mathrm{D}}{ }^{23}+83.6^{\circ}\left(c=0.59, \mathrm{CHCl}_{3}\right) ;{ }^{1} \mathrm{H} \mathrm{NMR}$ $\left(400 \mathrm{MHz}, \mathrm{CDCl}_{3}\right) \delta$ 7.35-7.20 (comp, 5H), 5.72 (dt, $\left.J=10.0,2.8 \mathrm{~Hz}, 1 \mathrm{H}\right), 5.01$ (dd, $J=$ 2.8, $1.8 \mathrm{~Hz}, 2 \mathrm{H}$ ), 4.96 (dt, $J=10.0,1.8 \mathrm{~Hz}, 1 \mathrm{H}$ ), 3.25 (fortuitous t, $J=9.0,7.7 \mathrm{~Hz}, 1 \mathrm{H}$ ), 2.14 (dd, $J=9.0,4.8 \mathrm{~Hz}), 1 \mathrm{H}), 1.56$ (dd, $J=7.7,4.8 \mathrm{~Hz}, 1 \mathrm{H}) ;{ }^{13} \mathrm{C}$ NMR $(100 \mathrm{MHz}$, $\left.\mathrm{CDCl}_{3}\right) \delta 171.9,135.5,129.1,128.4,127.2,124.7,121.2,69.2,35.2,26.8,22.7$; IR (neat) 3021, 2885, $1716 \mathrm{~cm}^{-1}$; HRMS (EI) calcd for $\mathrm{C}_{13} \mathrm{H}_{12} \mathrm{O}_{2} 200.0837$, found 200.0834 (M+).

3b: (6:1 hexanes/EtOAc, Rf = 0.36) 77\%, clear oil, GC: $(\mathrm{B}-\mathrm{DM}, 30 \mathrm{~m} \times 0.25 \mathrm{~mm} ; 0.25$ $\mu \mathrm{m}$ film, $160{ }^{\circ} \mathrm{C}$ isotherm) retention times of $82.4 \mathrm{~min}$ (minor) and $96.0 \mathrm{~min}$ (major), $80 \%$ ee; $[\alpha]_{\mathrm{D}}{ }^{21}+68.2^{\circ}\left(c=0.63, \mathrm{CHCl}_{3}\right) .{ }^{1} \mathrm{H}$ NMR $\left(400 \mathrm{MHz}, \mathrm{CDCl}_{3}\right) \delta 5.80(\mathrm{dt}, J=$ 10.2, $2.8 \mathrm{~Hz}, 1 \mathrm{H}), 5.57-5.42(\mathrm{~m}, 1 \mathrm{H}), 5.26$ (dt, $J=10.2,2.0 \mathrm{~Hz}, 1 \mathrm{H}), 5.01-4.99$ (comp, 2H), 2.47-2.43 (m, 1H), 2.10-1.45 (comp, 9H), 1.27 (dd, $J=7.4,4.4 \mathrm{~Hz}, 1 \mathrm{H}) ;{ }^{13} \mathrm{C}$ NMR $\left(100 \mathrm{MHz}, \mathrm{CDCl}_{3}\right) \delta 172.5,133.0,125.1,125.0,120.6,69.1,37.5,29.5,25.2,25.1,22.7$, 22.31, 22.25; IR (neat) 3020, 2879, $1716 \mathrm{~cm}^{-1}$; HRMS (EI) calcd for $\mathrm{C}_{13} \mathrm{H}_{16} \mathrm{O}_{2} 204.1150$, found $204.1148\left(\mathrm{M}^{+}\right)$.

3c: (6:1 hexanes/EtOAc, $\mathrm{Rf}=0.29)$ 81\%, clear oil, 8:1 mixture E,Z-3c diastereomers. Diastereomeric ratio determined by ${ }^{1} \mathrm{H}$ NMR prior to chromatography $(E-3 c 6.38 \mathrm{ppm}, Z$ 3c $6.47 \mathrm{ppm})$. Isolated yield of $E, Z-3 c$ obtained upon initial column chromatography (6:1 hexanes/EtOAc). Diastereomers isolated by further silica gel column chromatography ( $E$ 3c $\mathrm{Rf}=0.44$, dichloromethane).

E-3c: Clear oil, HPLC: (AD-H, 98:2 hexanes/2-propanol, $1 \mathrm{~mL} / \mathrm{min}$ ) retention times of $11.8 \mathrm{~min}$ (major) and $13.2 \mathrm{~min}$ (minor), $86 \%$ ee; $[\alpha]_{\mathrm{D}}{ }^{21}+77.8^{\circ}\left(c=0.73, \mathrm{CHCl}_{3}\right) ;{ }^{1} \mathrm{H}$ 
NMR (400 MHz, CDCl $) \delta$ 7.36-7.23 (comp, 5H), 6.38 (s, $1 \mathrm{H}$ ), 5.84 (dt, $J=10.2,2.8 \mathrm{~Hz}$, $1 \mathrm{H}$ ), 5.33 (br d, $J=10.2 \mathrm{~Hz}, 1 \mathrm{H}$ ), 5.05-5.03 (comp, 2H), 2.72 (fortuitous t, $J=7.9 \mathrm{~Hz}$, $1 \mathrm{H}), 1.96$ (dd, $J=8.7,4.7 \mathrm{~Hz}, 1 \mathrm{H}), 1.91(\mathrm{~s}, 3 \mathrm{H}), 1.47$ (dd, $J=7.4,4.7 \mathrm{~Hz}, 1 \mathrm{H}) ;{ }^{13} \mathrm{C}$ NMR $\left(100 \mathrm{MHz}, \mathrm{CDCl}_{3}\right) \delta 172.2,137.1,133.6,128.8,128.2,128.0,126.6,124.6,121.2,69.1$, 39.7, 25.8, 23.1, 19.1; IR (neat) 3050, 2884, $1722 \mathrm{~cm}^{-1}$; HRMS (EI) calcd for $\mathrm{C}_{16} \mathrm{H}_{16} \mathrm{O}_{2}$ 240.1150, found 204.1141 $\left(\mathrm{M}^{+}\right)$.

3d: (6:1 hexanes/EtOAc, $\mathrm{Rf}=0.33)$ 86\%, clear oil, 5:1 mixture of $E, Z$-3d diastereomers. Diastereomeric ratio determined by ${ }^{1} \mathrm{H}$ NMR prior to chromatography $(E-3 \mathbf{d} 1.28 \mathrm{ppm}$, Z-3d $1.45 \mathrm{ppm})$. Isolated yield of E,Z-3d obtained upon initial column chromatography (6:1 hexanes/EtOAc). Diastereomers separated by further chromatography $(E-3 \mathbf{d} \mathrm{Rf}=$ $0.54, Z$-3d $\mathrm{Rf}=0.25$, dichloromethane).

Z-3d: Clear oil, ${ }^{1} \mathrm{H}$ NMR (400 MHz, $\mathrm{CDCl}_{3}$ ) $\delta$ 7.36-7.19 (comp, 5H), 6.59 (d, $J=15.9$ Hz, 1H), 6.34 (dd, $J=15.9,9.1 \mathrm{~Hz}, 1 \mathrm{H}$ ), 5.84 (ddd, $J=9.9$, 3.6, $2.4 \mathrm{~Hz}, 1 \mathrm{H}$ ), 5.31 (ddd, $J$ = 9.9, 2.4, $1.2 \mathrm{~Hz}, 1 \mathrm{H}), 5.03(\mathrm{dt}, J=16.5,2.4,1 \mathrm{H}), 4.95$ (ddd, $J=16.5,3.6,1.2 \mathrm{~Hz}, 1 \mathrm{H}$ ), 2.16-2.10 (m, 1H), 2.05 (dd, $J=7.6,4.8 \mathrm{~Hz}, 1 \mathrm{H}), 1.45$ (dd, $J=8.5,4.8 \mathrm{~Hz}, 1 \mathrm{H}) ;{ }^{13} \mathrm{C}$ NMR $\left(100 \mathrm{MHz}, \mathrm{CDCl}_{3}\right) \delta 169.3,136.9,132.3,128.7,128.5,127.3,126.10,126.06$, 120.8, 69.1, 36.6, 28.8, 24.9; IR (neat) 3020, $1722 \mathrm{~cm}^{-1}$; HRMS (EI) calcd for $\mathrm{C}_{15} \mathrm{H}_{14} \mathrm{O}_{2}$ 226.0994, found 226.0989 $\left(\mathrm{M}^{+}\right)$.

E-3d: Clear oil, HPLC (OD-H, 90:10 hexanes/2-propanol, $1 \mathrm{~mL} / \mathrm{min}$ ) retention times of 15.0 min (minor) and 18.9 min (major), $73 \%$ ee; $[\alpha]_{\mathrm{D}}{ }^{22}+59.3^{\circ}\left(c=0.69, \mathrm{CHCl}_{3}\right) .{ }^{1} \mathrm{H}$ NMR (400 MHz, CDCl $) \delta$ 7.36-7.21 (comp, 5H), 6.60 (d, $J=15.8 \mathrm{~Hz}, 1 \mathrm{H}$ ), 5.96 (dd, $J$ = 15.8, 8.3 Hz, 1H), 5.86 (dt, $J=10.0,2.9,1 \mathrm{H}) 5.46(\mathrm{dt}, J=10.0,2.1 \mathrm{~Hz}, 1 \mathrm{H}), 5.02-5.01$ (comp, 2H), 2.76-2.70 (m, $1 \mathrm{H}$ ), 2.11 (dd, $J=8.9,4.6 \mathrm{~Hz}, 1 \mathrm{H}), 1.28$ (dd, $J=7.3,4.6 \mathrm{~Hz}$, $1 \mathrm{H}) ;{ }^{13} \mathrm{C}$ NMR $\left(100 \mathrm{MHz}, \mathrm{CDCl}_{3}\right) \delta$ 171.6, 136.6, 133.7, 128.6, 127.6, 126.0, 125.7, 125.9, 121.7, 69.1, 34.4, 27.4, 25.0; IR (neat) 3024, $1721 \mathrm{~cm}^{-1}$; HRMS (EI) calcd for $\mathrm{C}_{15} \mathrm{H}_{14} \mathrm{O}_{2}$ 226.0994, found $226.0989\left(\mathrm{M}^{+}\right)$.

Reduction and Cope rearrangement of 3d: Unseparated E,Z- 3d (5:1 E:Z) (203 mg, $0.90 \mathrm{mmol}$ ) was stirred in anhydrous THF $(10 \mathrm{~mL})$ at $0{ }^{\circ} \mathrm{C}$. $\mathrm{LiAlH}_{4}(93 \mathrm{mg}, 2.7 \mathrm{mmol})$ was added portionwise and the reaction mixture was heated to reflux under nitrogen. After $36 \mathrm{~h}$ the solution was cooled to $0{ }^{\circ} \mathrm{C}$ and quenched via dropwise addition of water until gas was no longer observed evolving. An aqueous solution of $\mathrm{NaOH}(0.5 \mathrm{~mL}, 50$ wt\%) was added, and the mixture was allowed to stir for 10 min. Anhydrous $\mathrm{Na}_{2} \mathrm{SO}_{4}$ was added and the mixture stirred $2 \mathrm{~h}$. The resulting aluminium salts were removed by filtration through Celite, washing with $\mathrm{Et}_{2} \mathrm{O}$ five times $(20 \mathrm{~mL})$. Solvent was removed from the filtrate by rotary evaporation, and the residue was recrystallized from a minimal amount of dichloromethane and hexanes to yield 4 (110 mg, 53\%, 92\% ee) as a white crystalline solid: $\mathrm{mp} 114{ }^{\circ} \mathrm{C}$, GC: (B-DM, 30m x $0.25 \mathrm{~mm}$; $0.25 \mu \mathrm{m}$ film, $140{ }^{\circ} \mathrm{C} / 10 \mathrm{~min}$, $1{ }^{\circ} \mathrm{C} / \mathrm{min}$ ramp to $160{ }^{\circ} \mathrm{C}$ ) retention times of $68.7 \mathrm{~min}$ (minor) and $70.5 \mathrm{~min}$ (major), 92\% ee; $[\alpha]_{\mathrm{D}}{ }^{20}-60.6^{\circ}\left(c=0.34, \mathrm{CHCl}_{3}\right) ;{ }^{1} \mathrm{H}$ NMR $\left(400 \mathrm{MHz}, \mathrm{CDCl}_{3}\right) \delta$ 7.30-7.20 (comp, 5H), 5.80-5.73 (comp, 2H), 5.61-5.57 (m, 1H), 4.05 (s, 2H), 3.58-3.53 (m, 1H), 3.43 (dd, $J=$ $10.4,4.9 \mathrm{~Hz}, 1 \mathrm{H}$ ), 3.37 (dd, $J=10.4,6.2 \mathrm{~Hz}, 1 \mathrm{H}), 3.13-3.08$ (m, $1 \mathrm{H}), 2.95-2.89(\mathrm{~m}, 1 \mathrm{H})$, 2.77 (dd, $J=18.9,6.5 \mathrm{~Hz}, 1 \mathrm{H}), 1.99$ (br s, $1 \mathrm{H}$ ), 1.67 (br s, $1 \mathrm{H}) ;{ }^{13} \mathrm{C}$ NMR $(100 \mathrm{MHz}$, 
$\left.\mathrm{CDCl}_{3}\right) \delta 144.0,142.9,133.8,128.5,128.2,126.8,126.5,126.2,68.0,65.0,45.6,45.0$, 28.6; IR (neat) $3020 \mathrm{~cm}^{-1}$.

Determination of absolute stereochemistry of 2a: A sample of 2a,b (9:1, $84 \mathrm{mg}, 0.48$ mmol) obtained from the $\mathrm{Rh}_{2}(4 S, R$-MenthAZ) catalyzed $\mathrm{C}-\mathrm{H}$ insertion $(80 \%$ ee as measured by GC) was stirred in toluene $(10 \mathrm{~mL})$. 2,3-Dichloro-5.6-dicyano-1,4benzoquinone (216 mg, $0.96 \mathrm{mmol}$ ) was added. After $2 \mathrm{~h}$, the toluene was evaporated and the reaction mixture was quickly passed through a short silica gel plug (3:1 hexane/ethyl acetate) to remove a bright red colored baseline impurity. The resulting solution was evaporated to a yellow oil. Ethyl acetate $(5 \mathrm{~mL})$ was added, followed by $10 \% \mathrm{Pd} / \mathrm{C}(10 \mathrm{mg})$. The atmosphere was purged and replaced with hydrogen. After stirring $12 \mathrm{~h}$, the mixture was filtered through a celite pad, washing with ethyl acetate, and evaporated. Column chromatography $(4: 1$ hexane:ethyl acetate, $\mathrm{Rf}=0.30)$ provided 2-phenyl- $\delta$-valerolactone $\left(71 \mathrm{mg}, 86 \%\right.$ yield). ${ }^{4}$ The optical rotation of 2-phenyl- $\delta$ valerolactone was measured: $[\alpha]_{\mathrm{D}}{ }^{23}+14.1^{0}\left(c=0.28, \mathrm{CHCl}_{3}\right)$. A previous report lists the optical rotation of the $R$ enantiomer (72\% ee) to be: $[\alpha]_{\mathrm{D}}^{22}+32.6^{\circ}\left(c=0.424, \mathrm{CHCl}_{3}\right)$, indicating the absolute stereochemistry of the predominant enantiomer of $2 \mathbf{a}$ to be $R .^{5}$

\footnotetext{
${ }^{4}$ Betancourt de Perez, R. M.; Fuentes, L. M.; Larson, G. L.; Barnes, C. L.; Heeg, M. J. J. Org. Chem. 1986, 51, 2039.

${ }^{5}$ Nakamura, Y.; Takeuchi, S.; Ohgo, Y.; Yamaoka, M.; Yoshida, A.; Mikami, K. Tetrahedron 1999, 55, 4959.
} 


\section{Determination of relative stereochemistry of $2 b$, 3a:}

nOe experiments were performed on a Bruker AM-400 instrument using a Gauss1.1000 shape pulse and gradient selection. Observed correlations were used to assign the relative stereochemistry of $\mathbf{2 b}$, $\mathbf{3 a}$ as those shown.

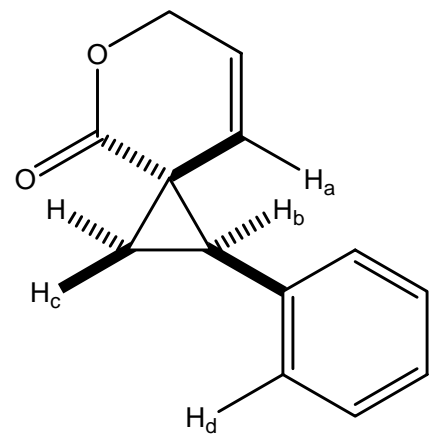

\begin{tabular}{|c|c|c|}
\hline $\begin{array}{l}\text { Irradiated Signal } \\
(\mathbf{p p m}) / \mathbf{H}_{\mathrm{x}}\end{array}$ & $\begin{array}{c}\text { Correlated Signals } \\
(\mathbf{p p m}) / \mathbf{H}_{\mathrm{x}}\end{array}$ & \% Correlation \\
\hline $4.96 / \mathrm{H}_{\mathrm{a}}$ & $\begin{array}{c}1.56 / \mathrm{H}_{\mathrm{c}} \\
7.21(\mathrm{~d}, J=7.3 \mathrm{~Hz}) / \mathrm{H}_{\mathrm{d}} \\
3.25 / \mathrm{H}_{\mathrm{b}}\end{array}$ & $\begin{array}{c}1.3 \\
0.8 \\
0\end{array}$ \\
\hline $3.25 / \mathrm{H}_{\mathrm{b}}$ & $7.21(\mathrm{~d}, J=7.3 \mathrm{~Hz}) / \mathrm{H}_{\mathrm{e}}$ & 1.1 \\
\hline $1.56 / \mathrm{H}_{\mathrm{c}}$ & $\begin{array}{c}4.96 / \mathrm{H}_{\mathrm{a}} \\
7.21(\mathrm{~d}, J=7.3 \mathrm{~Hz}) / \mathrm{H}_{\mathrm{d}}\end{array}$ & $\begin{array}{l}0.9 \\
2.1\end{array}$ \\
\hline
\end{tabular}




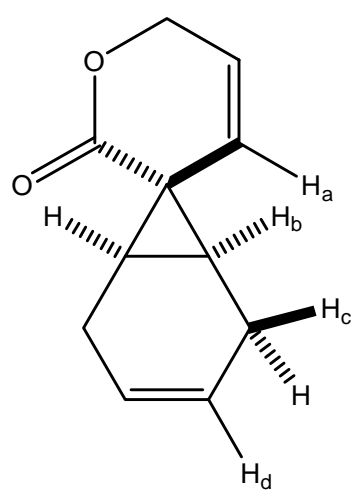

\begin{tabular}{|c|c|c|}
\hline $\begin{array}{l}\text { Irradiated Signal } \\
(\mathrm{ppm}) / \mathbf{H}_{\mathrm{x}}\end{array}$ & $\begin{array}{l}\text { Correlated Signals } \\
(\mathrm{ppm}) / \mathbf{H}_{\mathbf{x}}\end{array}$ & \% Correlation \\
\hline $5.63 / \mathrm{H}_{\mathrm{d}}$ & $5.42 / \mathrm{H}_{\mathrm{a}}$ & 0.1 \\
\hline $5.42 / \mathrm{H}_{\mathrm{a}}$ & $\begin{array}{c}5.63 / \mathrm{H}_{\mathrm{d}} \\
2.09-2.01 / \mathrm{H}_{\mathrm{c}} \\
2.19-2.16 / \mathrm{H}_{\mathrm{b}}\end{array}$ & $\begin{array}{c}0.3 \\
1.1 \\
0\end{array}$ \\
\hline $2.09-2.01 / \mathrm{H}_{\mathrm{C}}$ & $5.42 / \mathrm{H}_{\mathrm{a}}$ & 0.7 \\
\hline
\end{tabular}



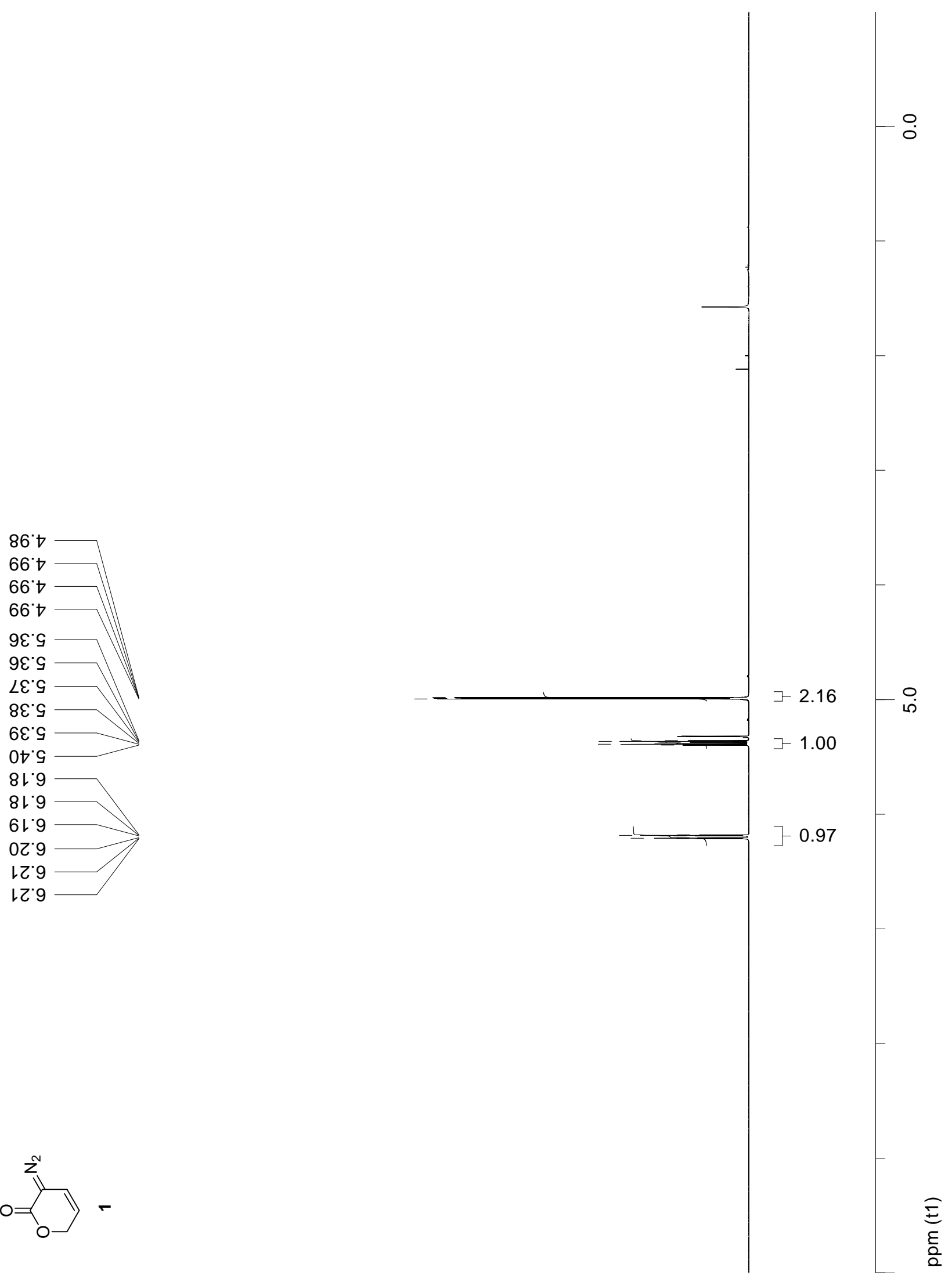

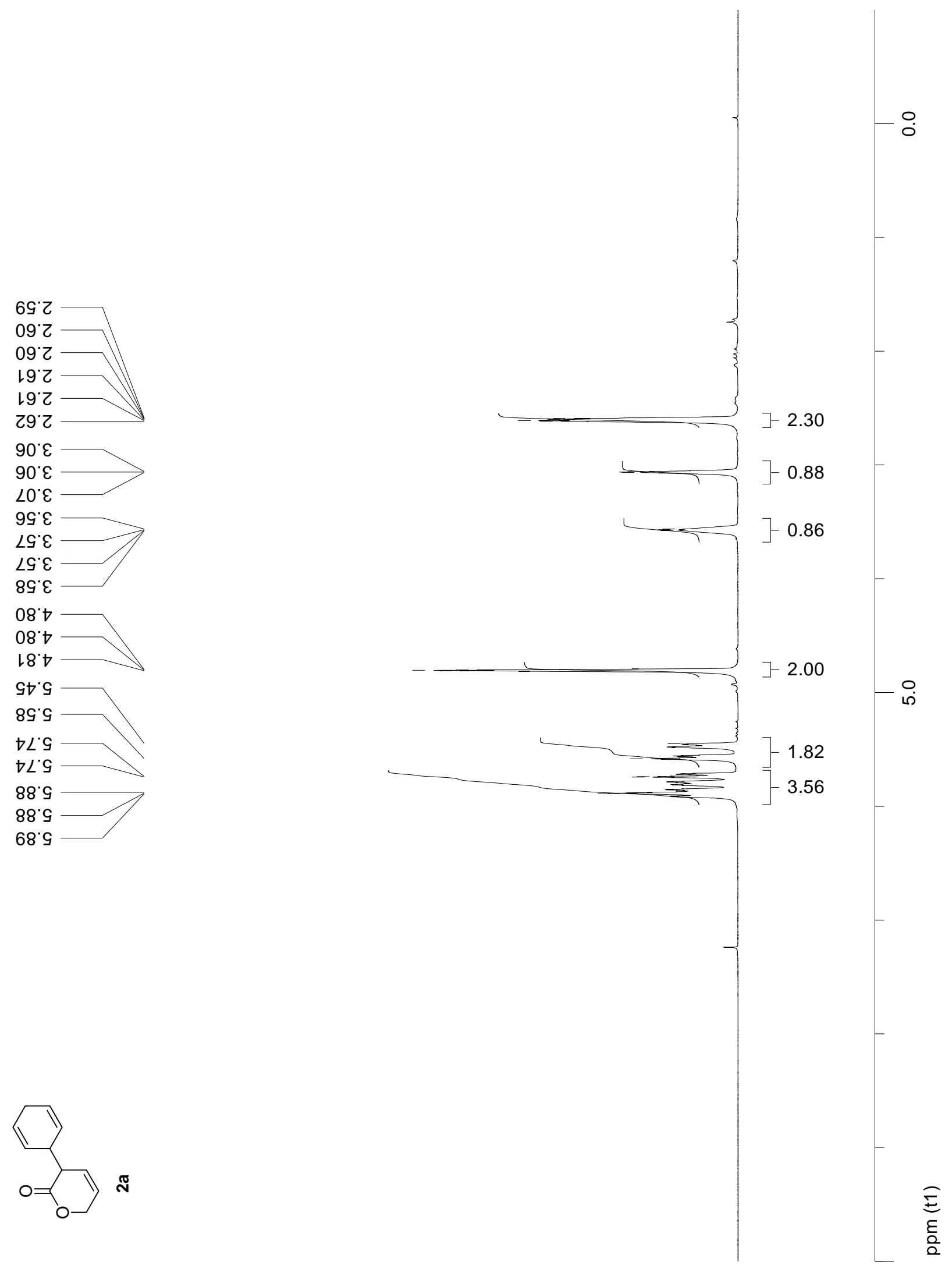

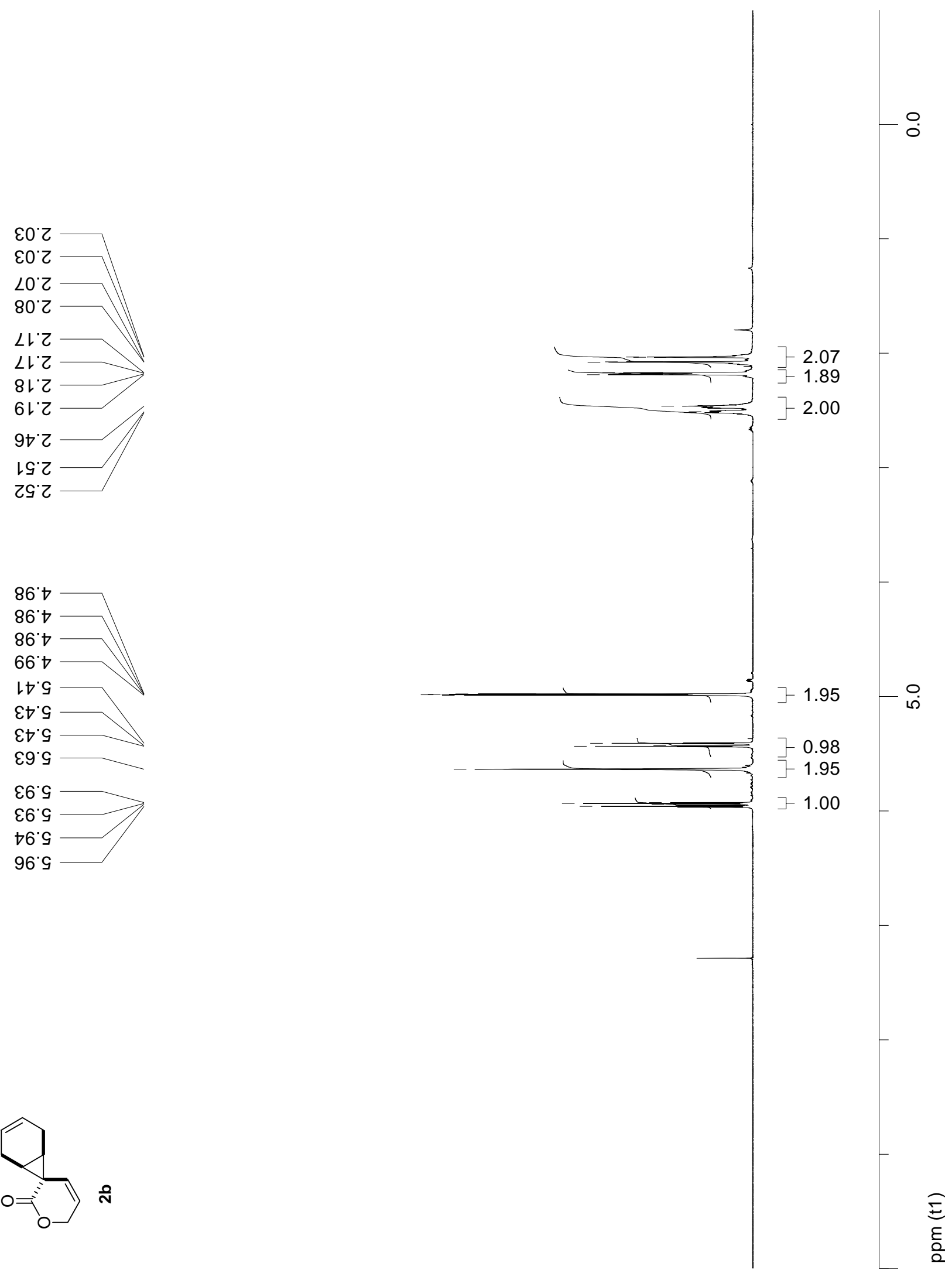

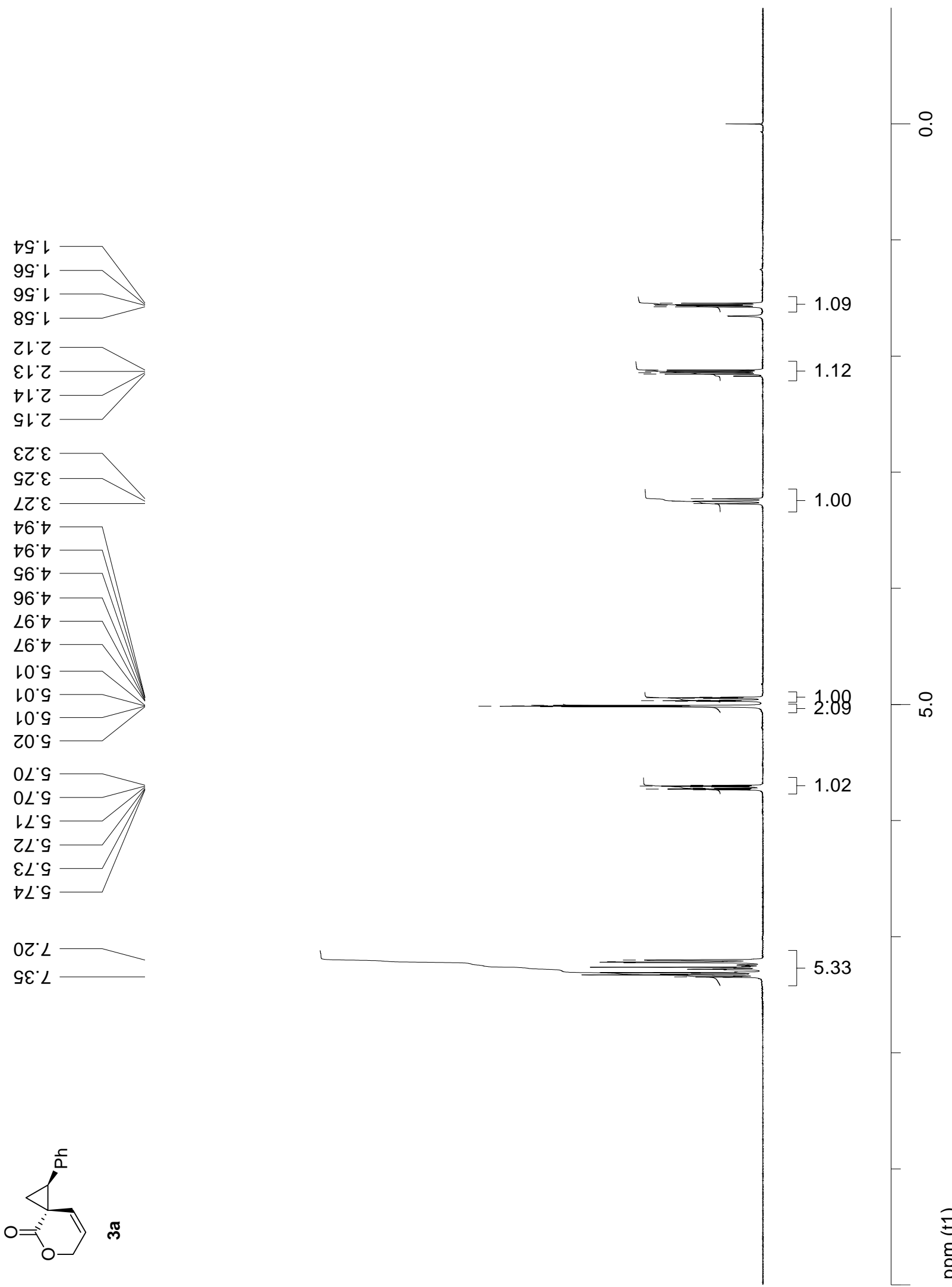

E
$\frac{\varepsilon}{2}$ 

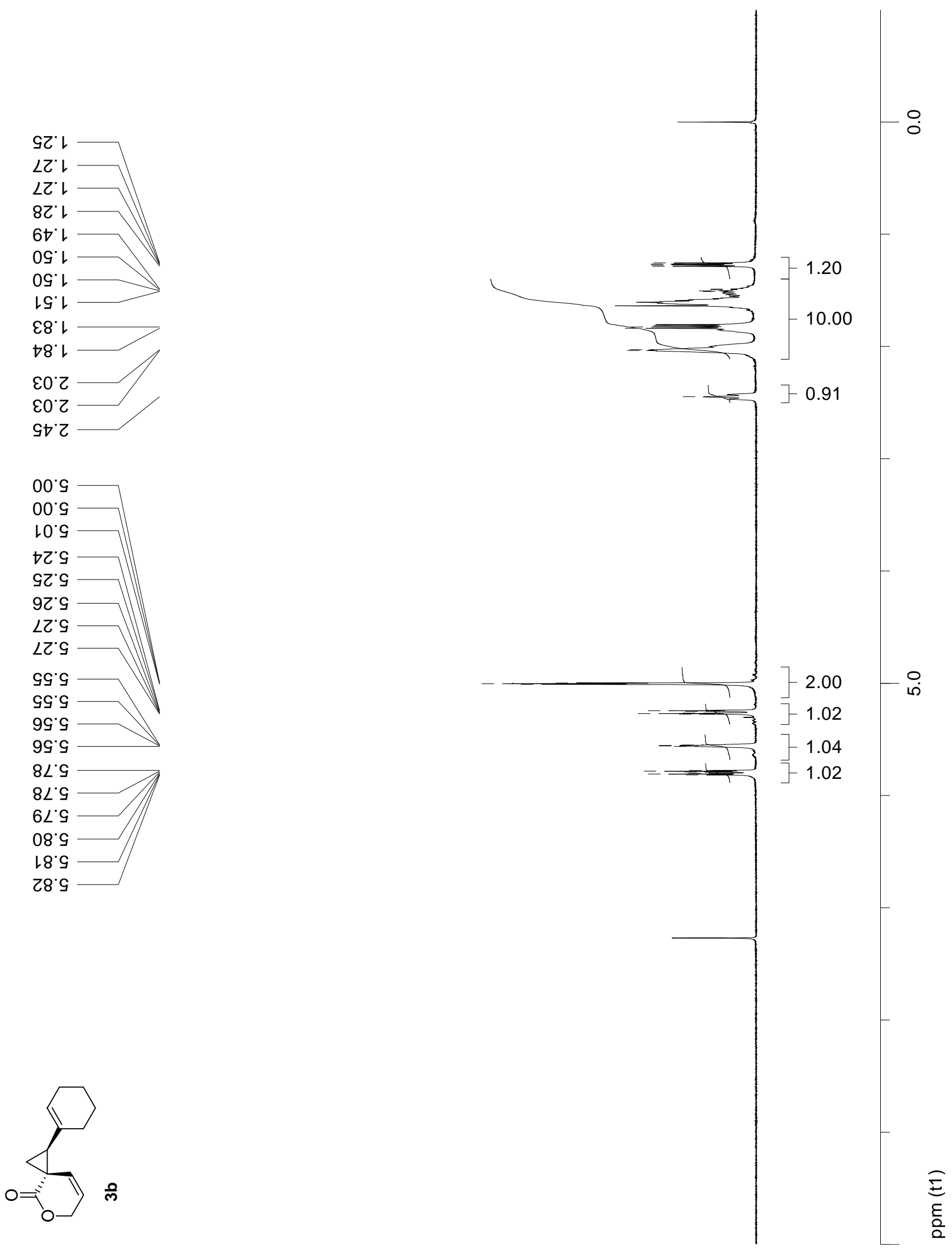

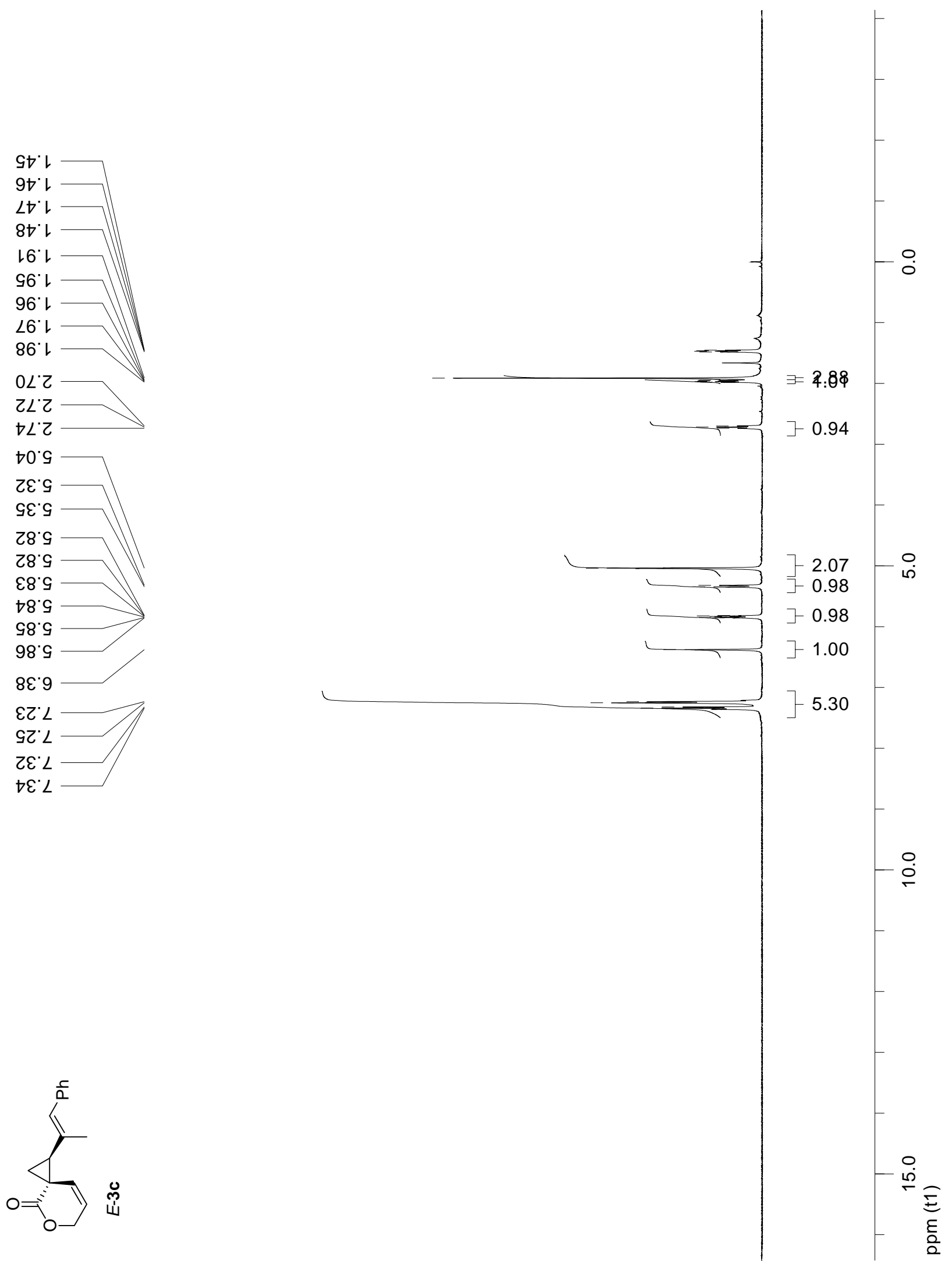

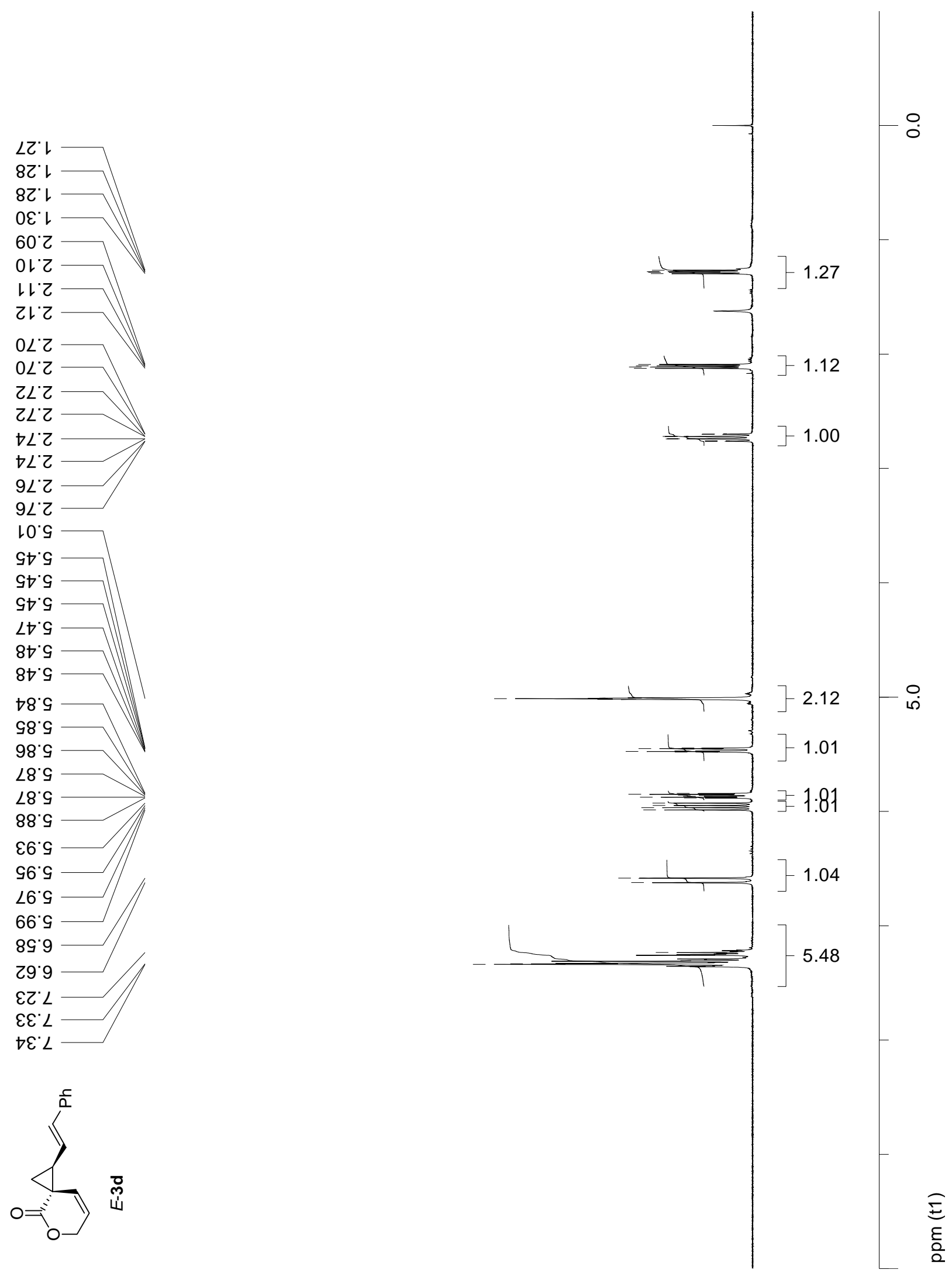

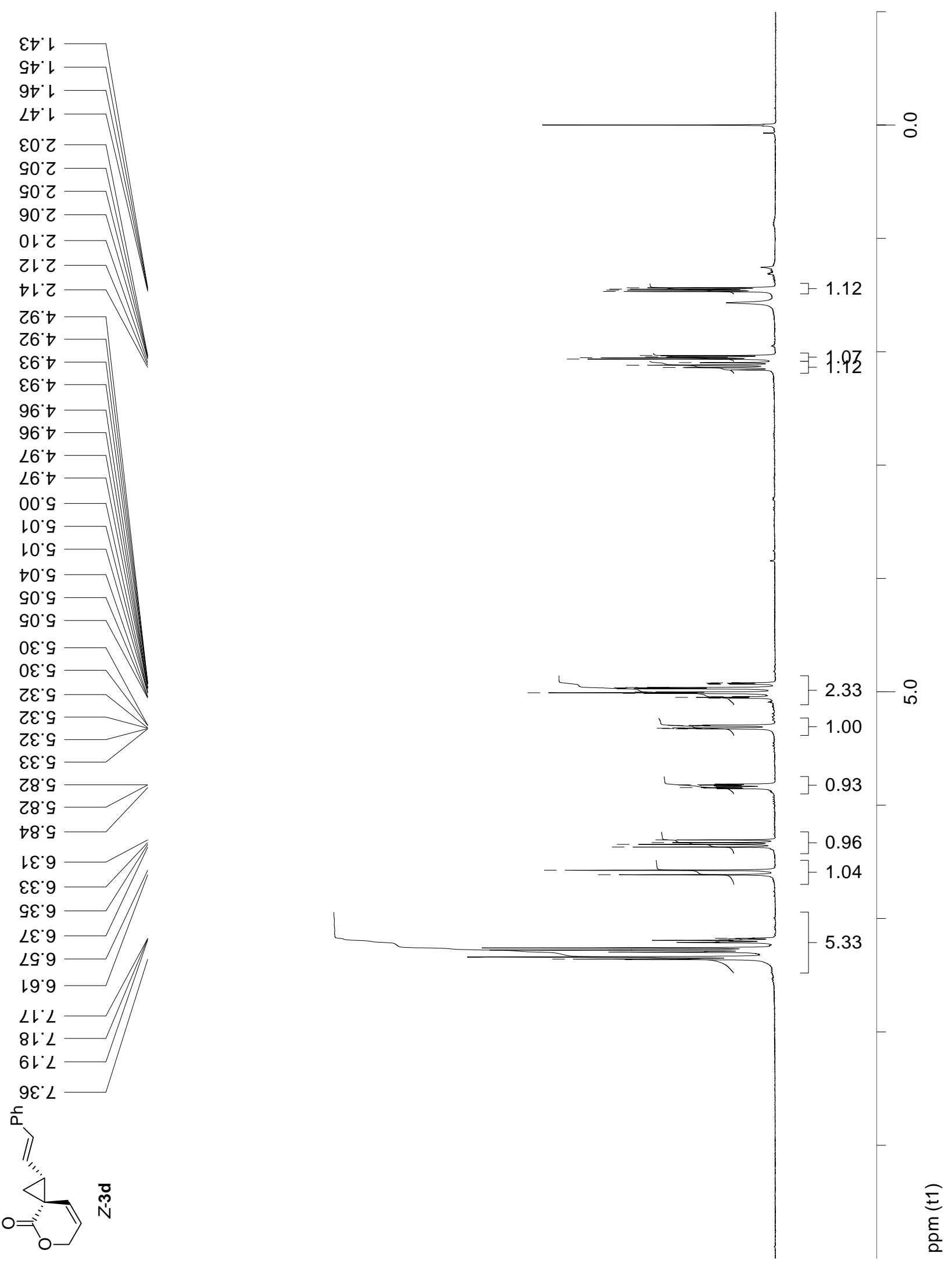

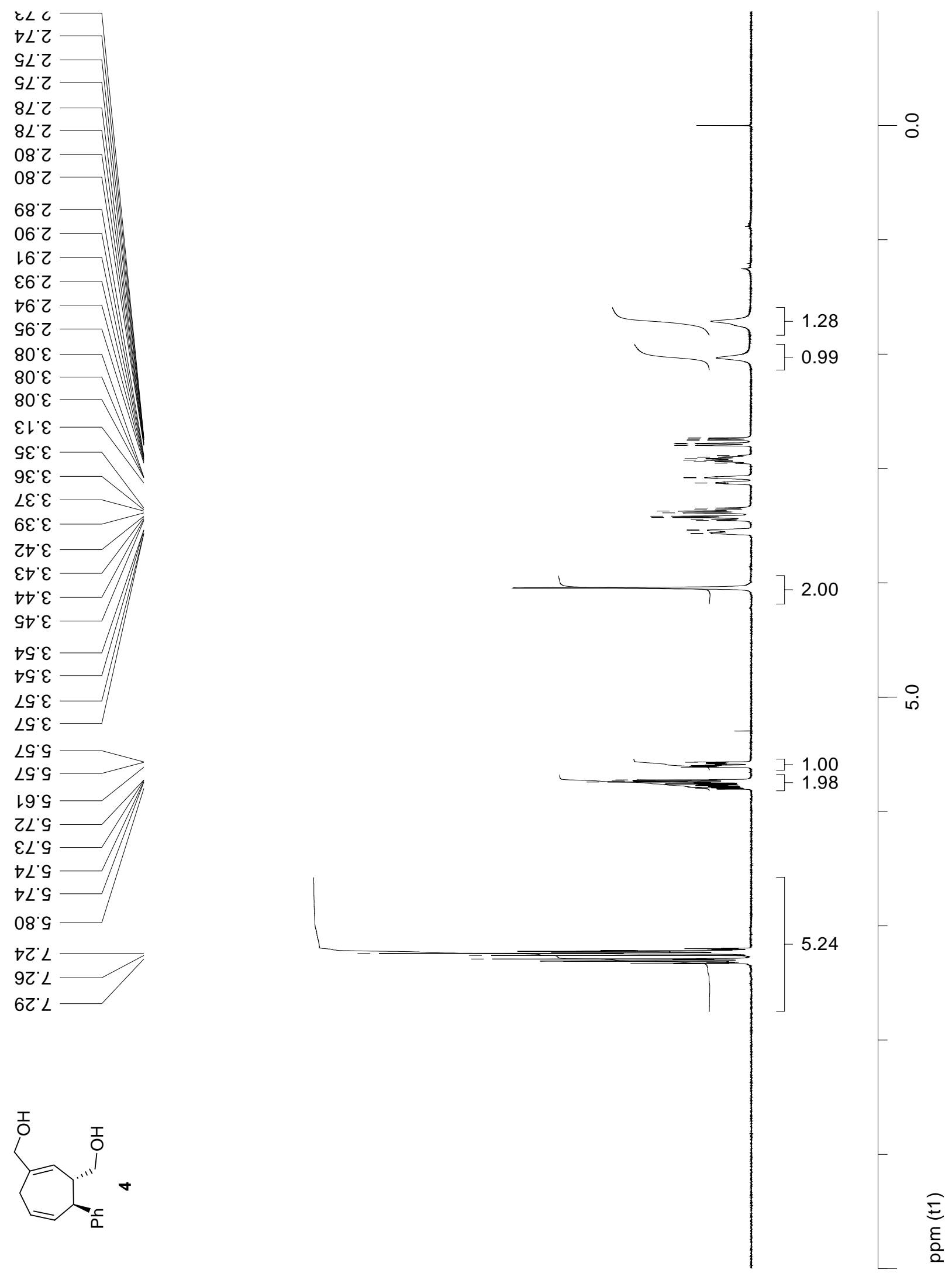


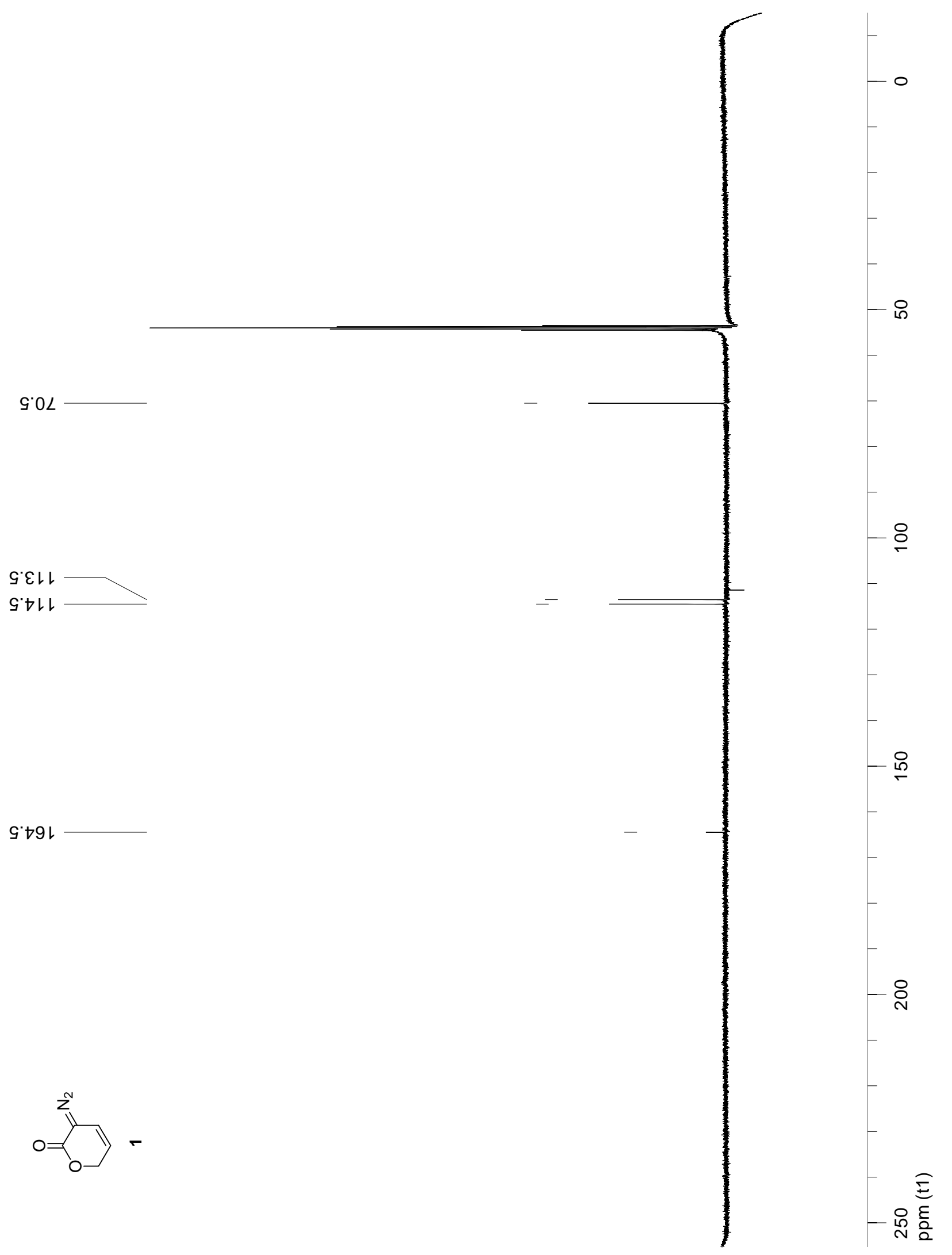




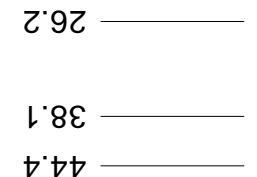

๑๋ヤ

8.89

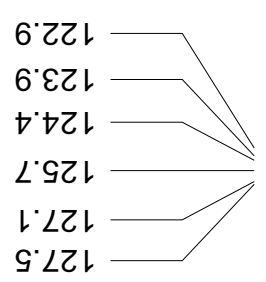

$9^{\circ} 0<\tau$

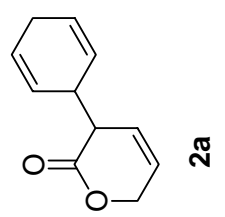

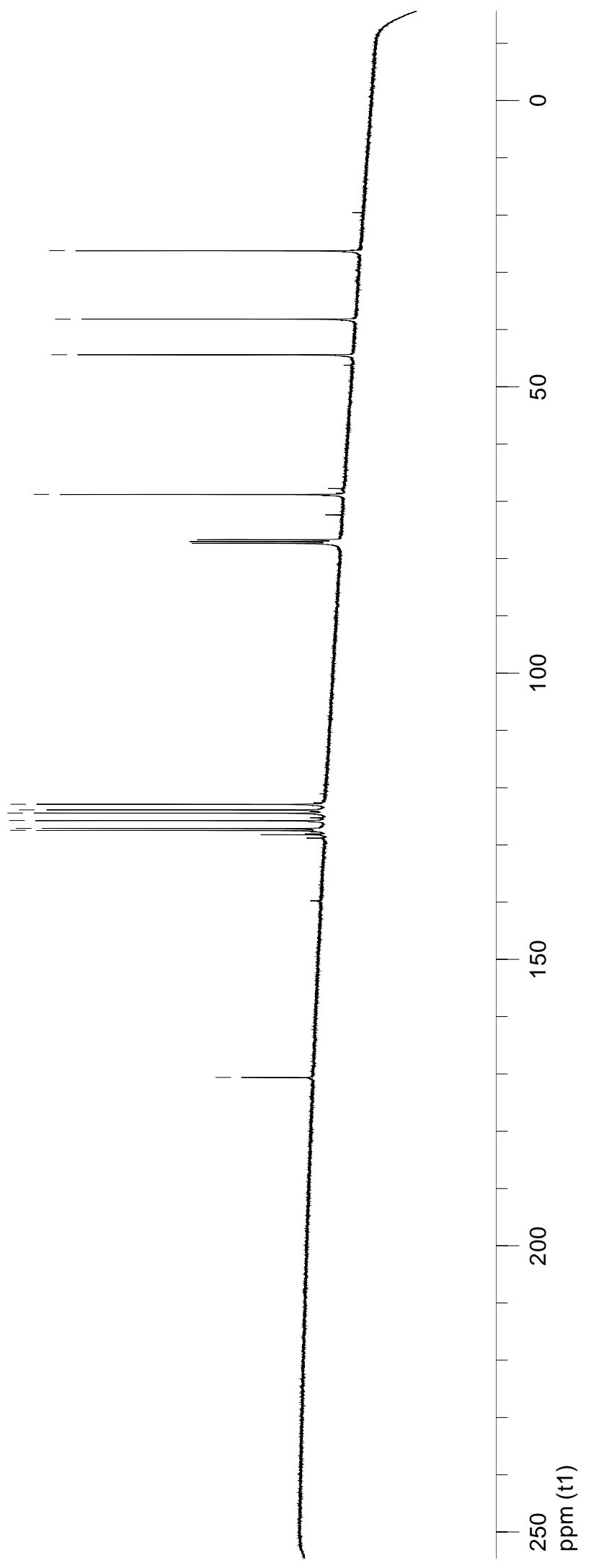


L'6

$\varepsilon \cdot 92$

Z. 82

L'89

ย'น乙โ

0 'ยटโ

$\varepsilon " \triangleright Z \tau$

$\angle ' Z L I$

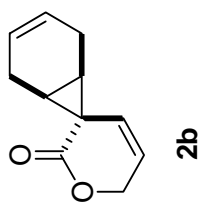

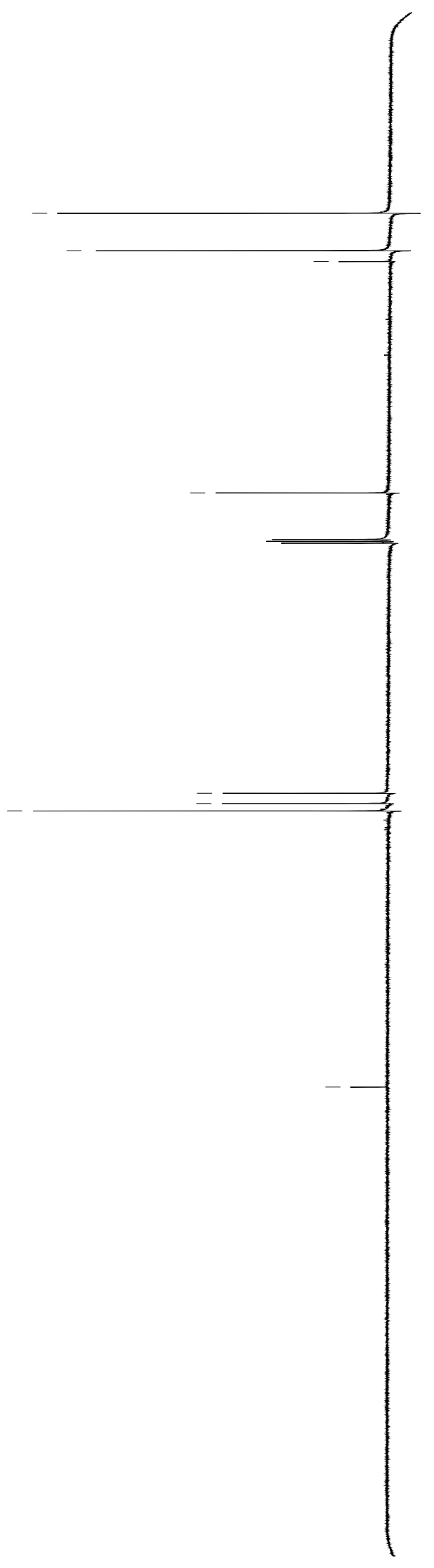

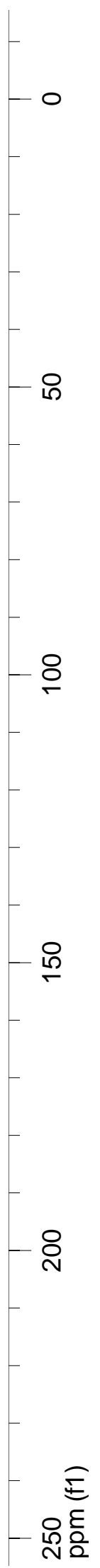




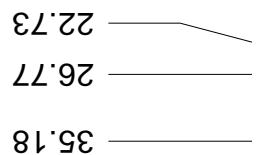

โZ'69

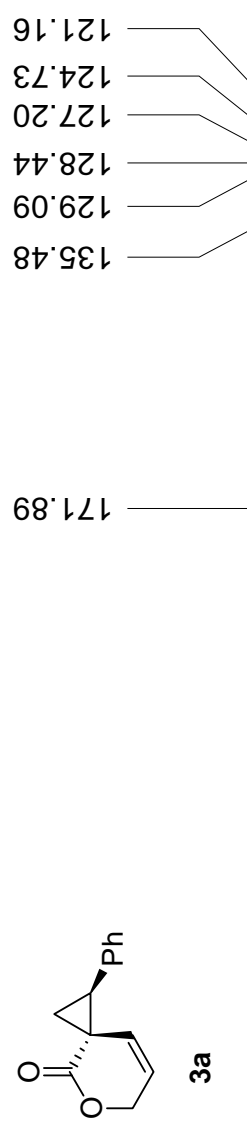

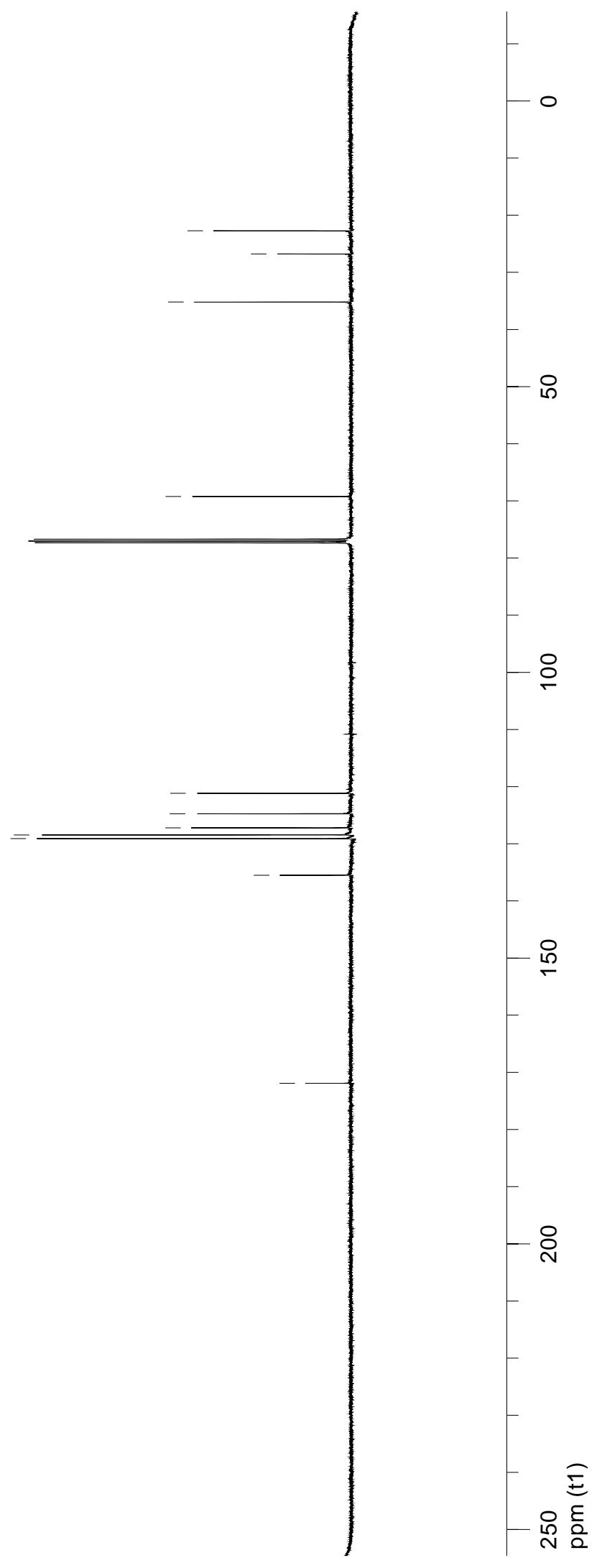




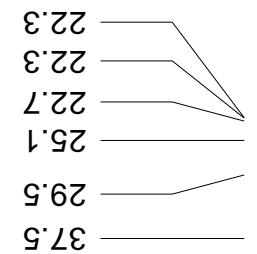

I'69

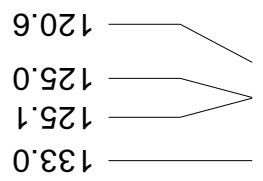

G'ZLI

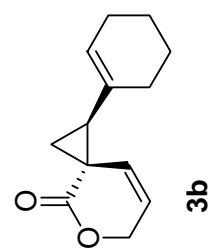

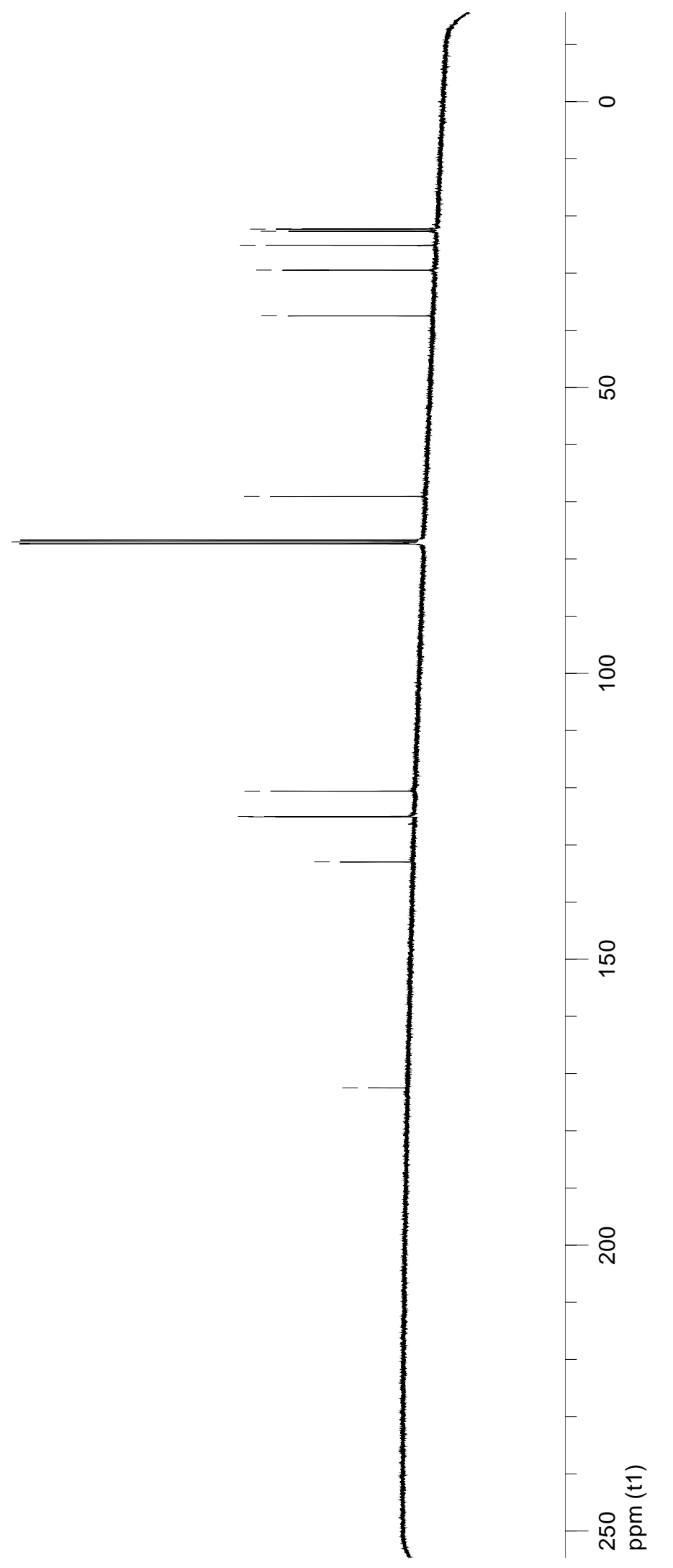




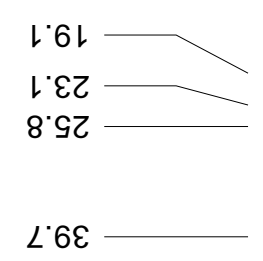

I'69

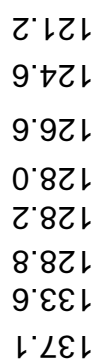

¿'ZL

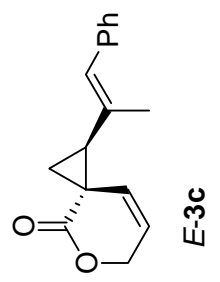

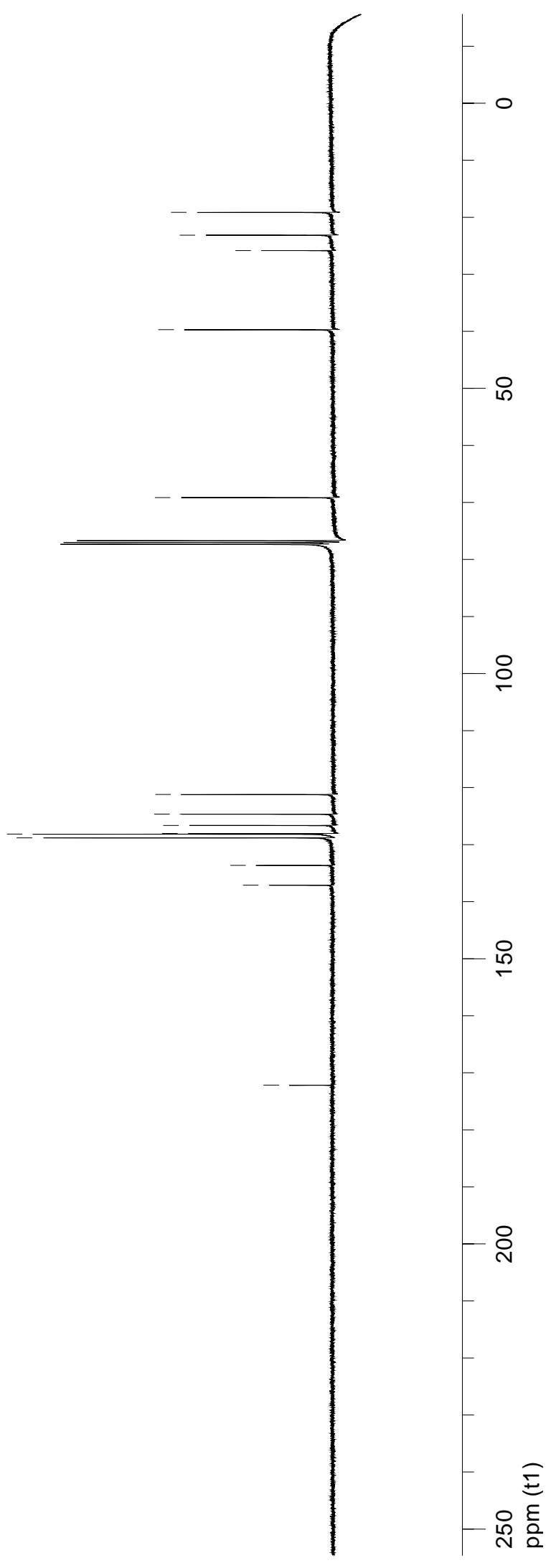


0.92

$\nabla^{\circ} L Z$

$\nabla^{*} \nabla \varepsilon$

โ:69

LTZT

6'ヤ乙二

LGZI

$0.92 \mathrm{~T}$

9 $\angle Z T$

9.8乙T

L'EEโ

9.9ع

$9^{\circ} T L T$

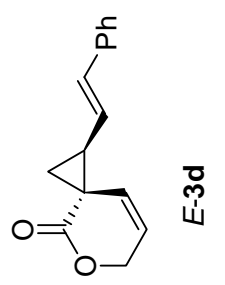

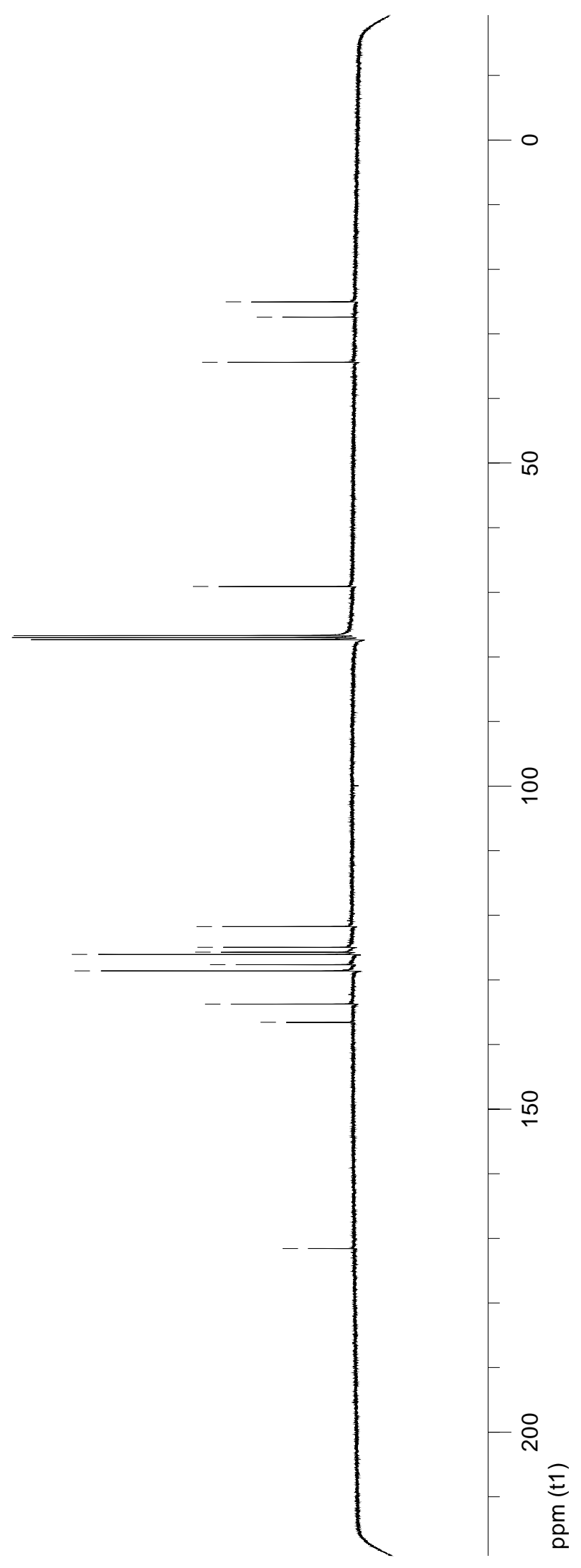


$6^{\circ} \nabla z$

8.82

$9 \cdot 9 \varepsilon$

I'69

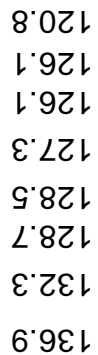

$\varepsilon^{\prime} 69 \tau$

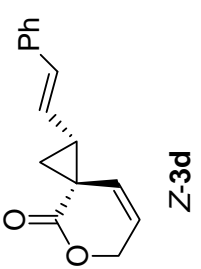

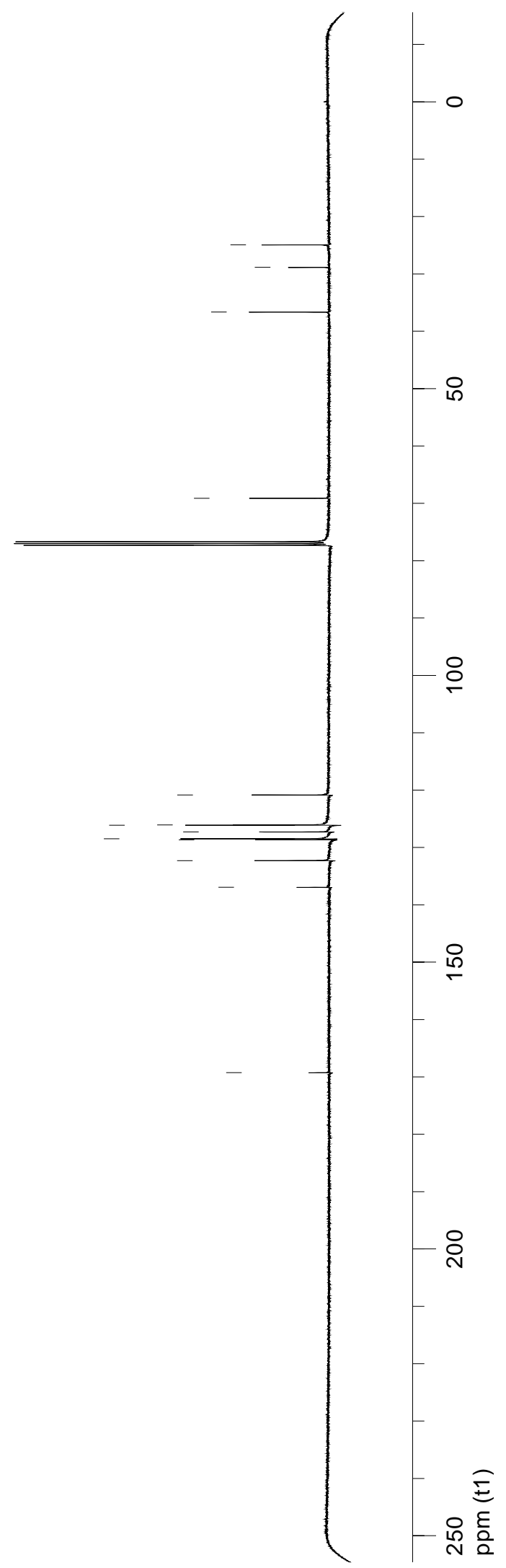



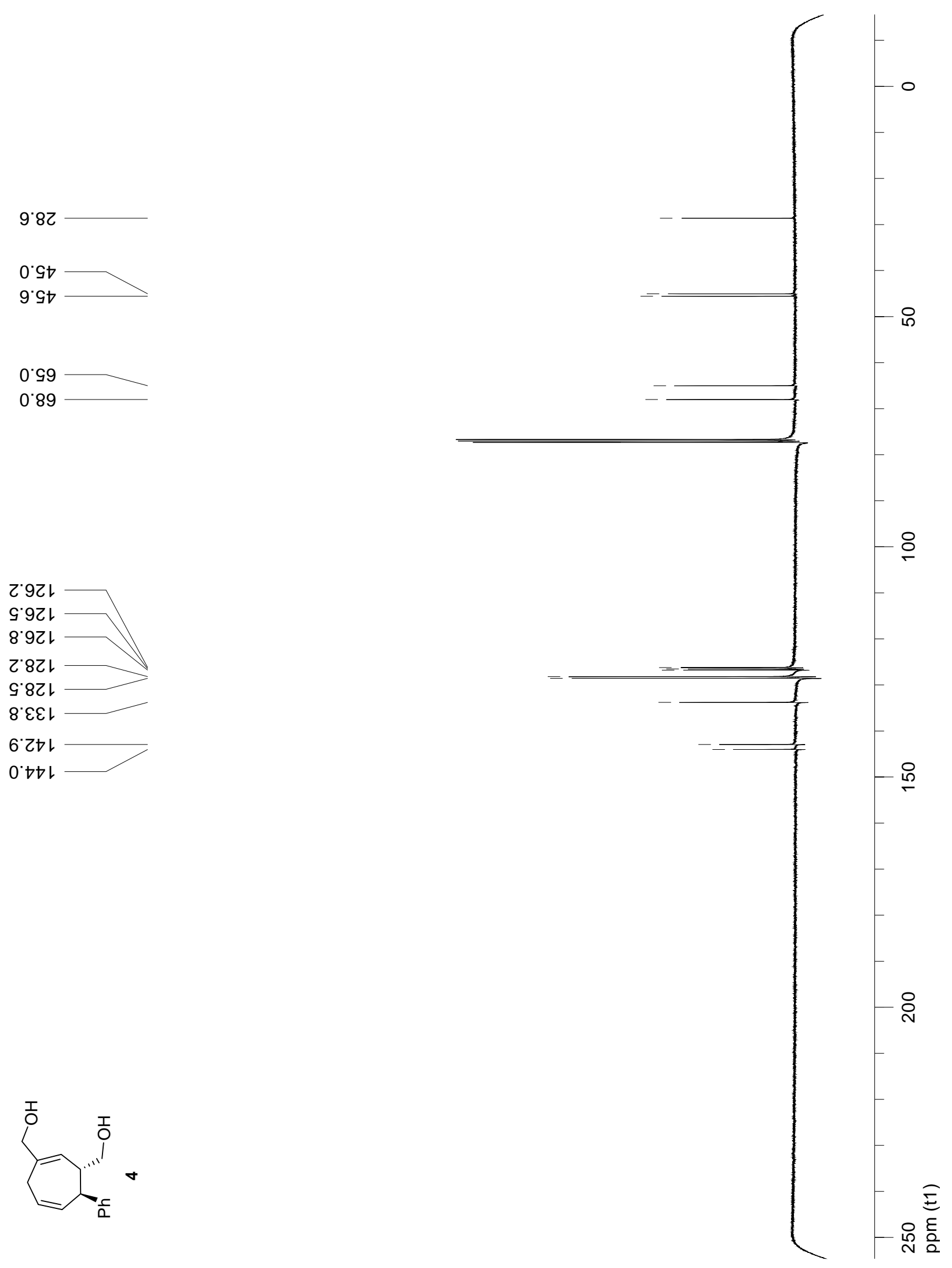


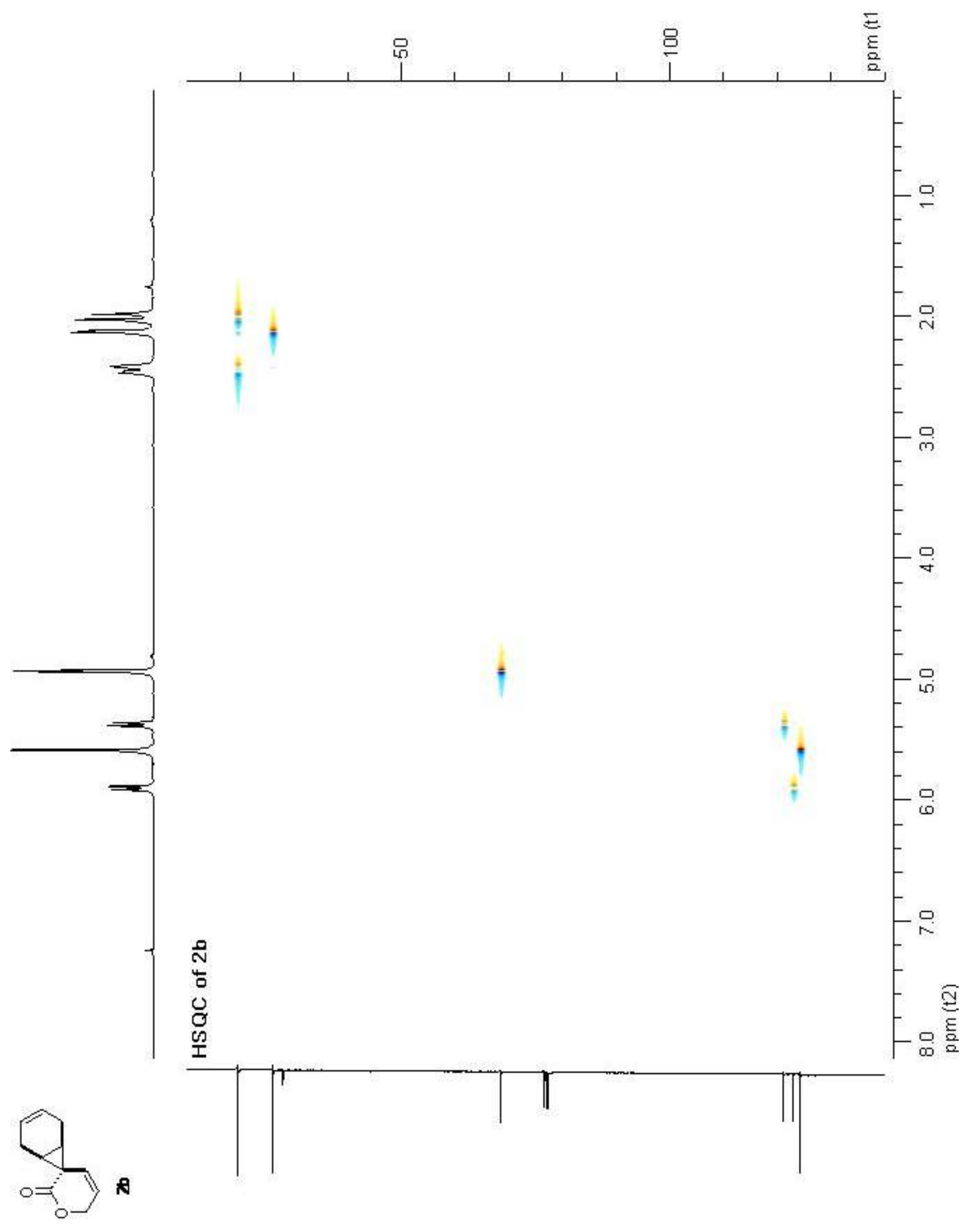

